\title{
THE NATO III 5 MHZ DISTRIBUTION SYSTEM
}

A. Vulcan and M. Bloch

(Frequency Electronics, Inc.), New Hyde Park, New York

\begin{abstract}
This paper describes a high performance $5 \mathrm{MHz}$ distribution system having extremely low phase noise and jitter characteristics and providing multiple buffered outputs. The system is completely redundant with automatic switchover and is self-testing. Faults can be isolated to a modular level by observing front panel status indicators. Since the $5 \mathrm{MHz}$ reference signals distributed by the NATO III distribution system are used for up-conversion and multiplicative functions, a high degree of phase stability and isolation between outputs is necessary. Unique circuit design and packaging concepts are utilized to insure that the isolation between outputs is sufficient to guarantee a phase perturbation of less than $0.0016^{\circ}$ when other outputs are open circuited, short circuited or terminated in $50 \mathrm{ohms.} \mathrm{The} \mathrm{circuit} \mathrm{design} \mathrm{techniques}$ include high isolation cascode amplifiers, the use of negative feedback to stabilize system gain and minimize circuit phase noise contributions, the use of balanced lines in lieu of single ended coaxial transmission media to minimize pickup and degradation of noise floor and the development of simplified fault detection and switchover circuitry to insure continuous operation.
\end{abstract}

The distribution system is fed by redundant high stability quartz frequency standards which use special crystals with low phase noise and jitter and a daily aging rate better than $5 \times 10^{-11}$.

\section{INTRODUCTION}

A $5 \mathrm{MHz}$ signal generation and distribution system is described which provides the basic reference frequencies for both a transportable and fixed satellite ground station. The system has extremely low vibration induced phase jitter and high isolation between outputs. Both of these characteristics are required for the NATO III satellite terminal mission. The system is modular and self-testing and any modules can be replaced without disturbing system operation. 


\section{SYSTEM DESCRIPTION}

The block diagram of Figure 1 shows how a dual frequency standard, Primary Distribution Unit (PDU) and Secondary Distribution Unit (SDU), are interconnected to provide a failsafe system having fifty-six $5 \mathrm{MHz}$ outputs, four $1 \mathrm{MHz}$ outputs and four $100 \mathrm{kHz}$ outputs. Two frequency standards in the Frequency Generation Unit (FGU) provide redundant, stable, low noise signals for the system input. The Electronic Switch Module (ESM) in the PDU accepts the $5 \mathrm{MHz}$ input and feeds it to two Primary Distribution Modules (PDM). The dual electronic switches provide redundancy in the event of faiure of either frequency standard, interconnecting cable, or ESM. The Primary Distribution Module receives the redundant $5 \mathrm{MHz}$ inputs and provides seven balanced outputs which feed various Secondary Distribution Units which can be located upto 500 feet from the PDU. Although the PDU, SDU and dual frequency standards comprise the NATO III station distribution system, the SDU can stand alone as a totally independent fifty-six channel low noise distribution system. Interconnection between the PDU and SDU is accomplished on a redundant basis such that the failure of either balanced interconnecting cable or PDM will not effect the final output signal. The requirement for low spurious signals and phase noise necessitates the use of double shielded RG-22B/U twinaxial 95 ohm balanced cable. The entire signal transmission system is isolated from the environmental ground reducing the pickup of unwanted signals due to ground loops and non-common mode noise. Table 1 lists the performance characteristics of the system and Figures 2 and 3 are the detailed block diagrams for the PDU and SDU, respectively.

An amplitude limiting circuit in the SDU Preamplifier Module (PAM) maintains a constant output level of $+5 \mathrm{dBm}$ from the SDU over a wide range of input signal levels. This is important so as not to affect the operation of user equipment when interconnecting cables are disconnected or a PDM module in the PDU is removed. Seven independent modules each having eight outputs comprise the balance of the SDU.

The equipment is designed to operate from American or European ac main voltages and frequencies. Dual power regulators ensure that $60 \mathrm{~Hz}$ and $120 \mathrm{~Hz}$ line related phase modulations are reduced to negligible levels. A high current regulator in each of the dual power supplies and an additional three-terminal device in each module provides $0.01 \%$ line regulation with 150 microvolts of noise. An additional benefit of this system is that radiated susceptibility of the equipment from pickup of extraneous signals from colocated high power $R F$ devices is minimized since the module regulators have the ability to reject interfering input signals by at least $80 \mathrm{~dB}$ from $30 \mathrm{~Hz}$ to $10 \mathrm{kHz}$ and $40 \mathrm{~dB}$ up to $300 \mathrm{kHz}$. Additional RF filtering is used to meet the EMC requirements up to 18 $\mathrm{GHz}$. 
Each active circuit which is critical to system operation is failuredetected. Diode detectors are used for RF alarm generation and micrologic comparators sense the degradation of signal level below a preset value. The alarm signals are summed in each particular subsystem and fed to a common point where an output is provided to external monitoring equipment. Additionally, front panel indicators on the various modules visually indicate the occurrence of a failure or out of spec condition.

The system is designed such that removal or replacement of any module can be effected without causing intolerable phase or amplitude perturbations on other active outputs. This design insures that $5 \mathrm{MHz}$ perturbations which can be multiplied 1000 to 2000 times in subsequent chains of frequency multiplications do not cause system outages. Minimal phase perturbations are insured by the use of high isolation amplifiers and a high degree of shielding between active circuitry.

\section{CIRCUIT DESIGN}

The following paragraphs describe two important functional blocks of the the distribution system.

\section{RF Amplifiers}

The amplifier stages used for $5 \mathrm{MHz}$ processing in various parts of the system are cascode circuits with high isolation, low noise, and high dynamic range. This circuit is shown schematically in Figure 4. To meet the isolation requirement of $100 \mathrm{~dB}$, special layout and packaging techniques were utilized. Circuit gain is controlled by the unbypassed emitter resistor which provides ac and dc stabilization of the circuit. Output transformation circuitry converts the collector impedance to 50 ohms with a source VSWR of 1.2 to 1 . Good grounding techniques with minimization of base lead inductance and parasitic reactances are necessary to insure optimal performance of the circuit. In the case where a balanced $95 \mathrm{ohm}$ output impedance is required, a small toroidal transformer is utilized to effect the impedance transformation.

Diode CR1 provides bias temperature compensation and CR2 is an RF detector. Capacitor $\mathrm{ClO}$ is selected for a detected output voltage of 0.25 volts at the nominal RF level. The dc voltage feeds a voltage comparator whose reference voltage is set to provide an alarm signal at a point approximately $1-1 / 2$ to $2 \mathrm{~dB}$ below the minimum acceptable $\mathrm{RF}$ leve1. Thus, temperature effects and other drift parameters will not cause a false alarm.

The various alarm signals are fed into the alarm module in the PDU and preamplifier module in the SDU to generate a composite alarm. These alarm signals which are TTL compatible, are summed with externally generated alarms which are translated to the proper levels and 
impedances before summation. In the case where the external alarm signals are fed from long twisted shielded pairs or are generated from relay contacts and/or noisy sources which may have high ac ground loop currents, optical couplers are used to isolate the noisy environment from the systems.

\section{Limiting}

The SDU must be capable of accepting an input dynamic range of $10 \mathrm{~dB}$ and maintain a constant output of $+5 \mathrm{dBm} \pm 1 \mathrm{~dB}$. Hence, a limiting circuit is necessary which has low noise characteristics and a constant input and output impedance to properly terminate the power splitters and maintain high isolation. Figure 5 shows the limiter used to achieve these objectives. Closely matched back-to-back $\mathrm{RF}$ signal diodes limit the $R F$ amplitude to +0.7 volts and the reflected power is absorbed in the hybrid terminations. The quadrature hybrids are implemented with lumped elements as shown in the figure.

\section{PHASE PERTURBATION MEASUREMENT}

Figure 6 is a block diagram showing how the 0.0016 degree $5 \mathrm{MHz}$ phase perturbation requirement is verified. The $5 \mathrm{MHz}$ input signal feeds two high isolation amplifiers. One amplifier output feeds a Xl000 multiplier chain and is used as the unperturbed reference. The other amplifier output feeds the unit under test which then drives an identical multiplier. The $5000 \mathrm{MHz}$ outputs are mixed to yield a dc signal where amplitude is proportional to the phase difference at the mixer inputs. The mixer output is amplified and fed to a high sensitivity oscilloscope. Prior to making a measurement, the variable phase shifter is adjusted for maximum outputs from the FE-6093A Microwave Test Set. This condition determines $E_{0}$ in the relationship $\Delta \phi=\sin ^{-1}\left(\Delta \mathrm{e} / \mathrm{E}_{0}\right) \quad \mathrm{X} \quad 10^{-3}$ where $\Delta \phi \quad$ is the $5 \mathrm{MHz}$ phase perturbation and $\Delta \mathrm{e}$ is the change in dc output voltage. The phase shifter is then adjusted for zero volts at the oscilloscope input (point of maximum mixer sensitivity), and the system is perturbed. The value of $\Delta \mathrm{e}$ is noted and $\Delta \phi$ is calculated. For example, if $\mathrm{E}_{0}$ is 1.50 volts and a $\Delta_{e}$ of 50 millivolts is recorded, $\Delta \phi$ equals 0.0015 degrees. This measurement technique is quite versatile and is used for measuring both steady state and transient phase shifts that occur from shock and vibration events, removing and replacing modules, changing load impedances, and varying ac voltage inputs.

\section{MECHANICAL PACKAGING}

A modular concept has been used for the frequency distribution subsystem in order to enhance maintainability, simplify logistics, and permit economic manufacturing. Figure 7 is a photograph of the FGU showing both quartz frequency standards and a phase comparator. Internal batteries are provided for 24 hours of operation without ac power. 
Figures 8 and 9 show the PDU which consists of five different module types. Each module is totally enclosed and has an RFI filter compartment in order to preserve shielding integrity. The modules can be inserted or removed directly from the front panel without disturbing other modules. Each module has a power indicator and various status and fault lights. Front panel test points are provided for monitoring the redundant dc voltages. Figures 10 and 11 shows the mechanical construction of the SDU. This drawer consists of three different module types, preamplifier, distribution amplifier and power supply. Seven distribution amplifiers are used to provide the fifty-six outputs. Figure 12 shows the internal construction of a preamplifier module. Each amplifier section is shielded to maintain an isolation of $100 \mathrm{~dB}$ between outputs. The RFI filtering compartment is totally isolated and the input connector directly addresses this compartment. Power, command and alarm signals are RFI filtered at this interface.

\section{ENVIRONMENTAL CONSIDERATIONS}

The frequency distribution subsystem has been designed to meet the environmental specification shown in Table 1 . Conservative component derating ensures that the equipment has a service life of 15 years. Performance specifications are met during high $G$ inputs specifically encountered in transportable vans. Care has been taken to insure that microphonically induced phase and amplitude modulations are reduced to negligible levels. Critical circuit elements are staked in place using resilient adhesives and all $\mathrm{RF}$ interconnection cables are likewise encapsulated. The PC Boards are coated with a humidity resistant material. The circuitry has been designed to be essentially broadband in nature to minimize the effect of temperature variations on output levels. Over a range of 0 to $+50^{\circ} \mathrm{C}$, the level variation is less than $\pm 0.2 \mathrm{~dB}$.

\section{CONCLUSION}

This paper describes a low noise frequency distribution system which is designed for continuous use in severe environments. The design stresses both long term reliability and electrical performance which emphasizes low spurious signals, low cross talk and low phase perturbations. The system consisting of a Frequency Generation Unit, a Primary Distribution Unit and a Secondary Distribution Unit has been qualified to MIL-E-16400 for environment and MIL-STD-461 for electromagnetic compatibility. 


\section{TABLE 1}

PERFORMANCE CHARACTERISTICS

FREQUENCY DISTRIBUTION SUBSYSTEM

1. OUTPUTS :

$$
\begin{array}{rr}
\text { FIFTY-SIX } & 5 \mathrm{MHz} \text { AT }+5 \mathrm{dBm} \\
\text { FOUR } & 1 \mathrm{MHz} \text { AT }+13 \mathrm{dBm} \\
\text { FOUR } & 100 \mathrm{KHz} \text { AT }+13 \mathrm{dBm} \\
\text { ONE } & 50 \mathrm{KHz} \text { AT }-100 \mathrm{dBm}
\end{array}
$$

2. IMPEDANCE :

$50 \Omega$ UNBALANCED, $95 \Omega$ BALANCED

3. VSWR:

1. $2: 1$

4. PHASE PERTURBATIONS: $0.0016^{\circ}$

5. AMPLITUDE

PERTURBATIONS :

$0.01 \mathrm{~dB}$

6. SPECTRAL PURITY:

- 120 dBC FROM $50 \mathrm{~Hz}$ TO 2 MHz OFFSET FROM CARRIER

7. PHASE NOISE:

2 dB ADDITIVE COMPONENT, - $165 \mathrm{dBc} / \mathrm{Hz}$ FLOOR

8. HARMONICS :

$40 \mathrm{~dB}$

9. FREQUENCY STABILITY: $1 \times 10^{-9} /$ MONTH, $2 \times 10^{-12} /$ SECOND

10. MICROPHONICS :

$1 \times 10^{-9} / \mathrm{G}$

11. MTBF :

$3.0,000$ HOURS

12. EMC:

MIL-STD-461

13. VIBRATION:

2.5G FROM $2 \mathrm{~Hz}$ TO $500 \mathrm{~Hz}$

14. SHOCK :

15G FOR 11 mECOND

15. HUMIDITY :

$95 \%$ RELATIVE

16. TEMPERATURE :

$-20^{\circ} \mathrm{C} \mathrm{TO}+65^{\circ} \mathrm{C}$ 


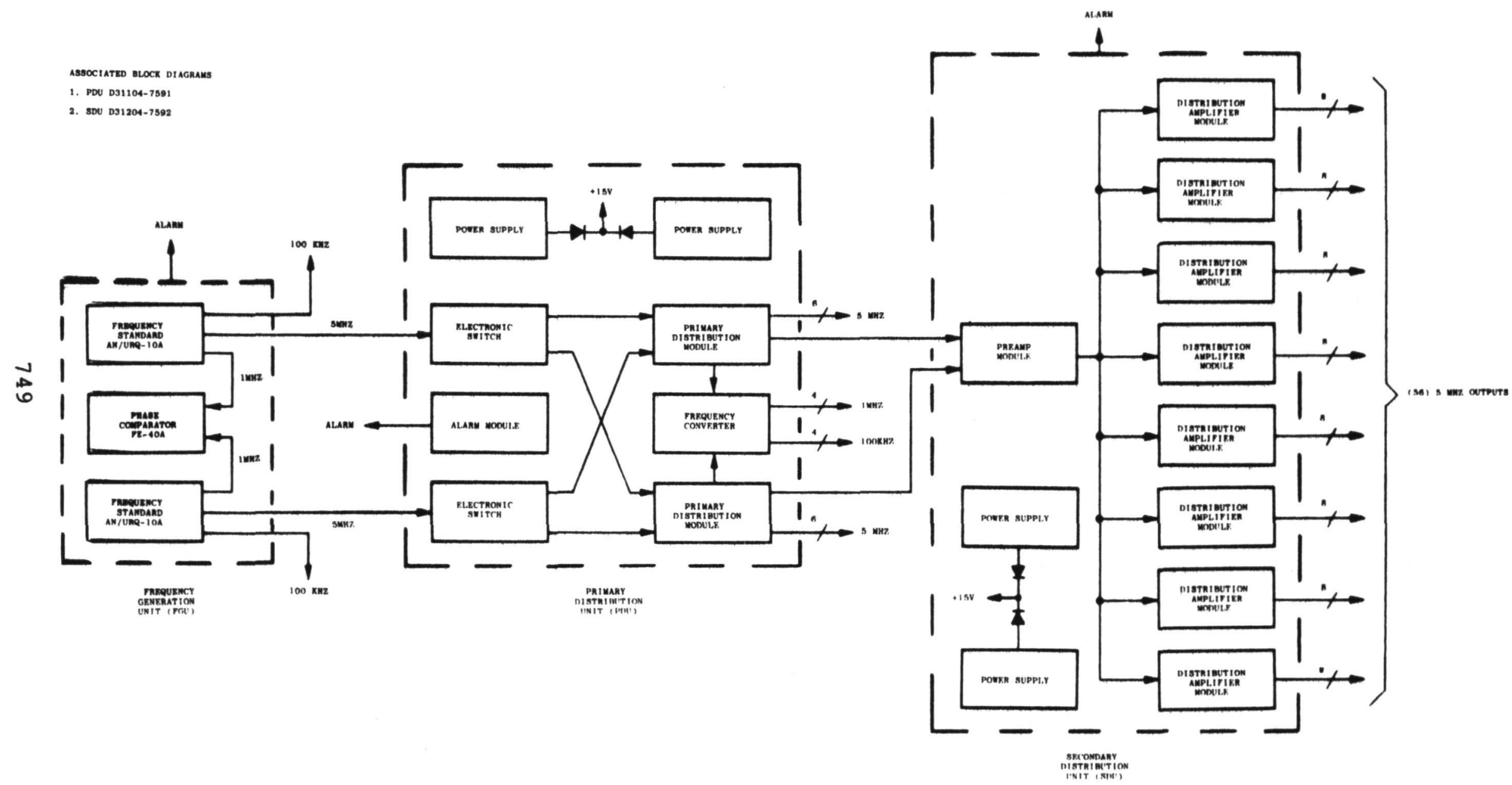

Figure 1. Frequency Distribution Sub-System Block Diagram 


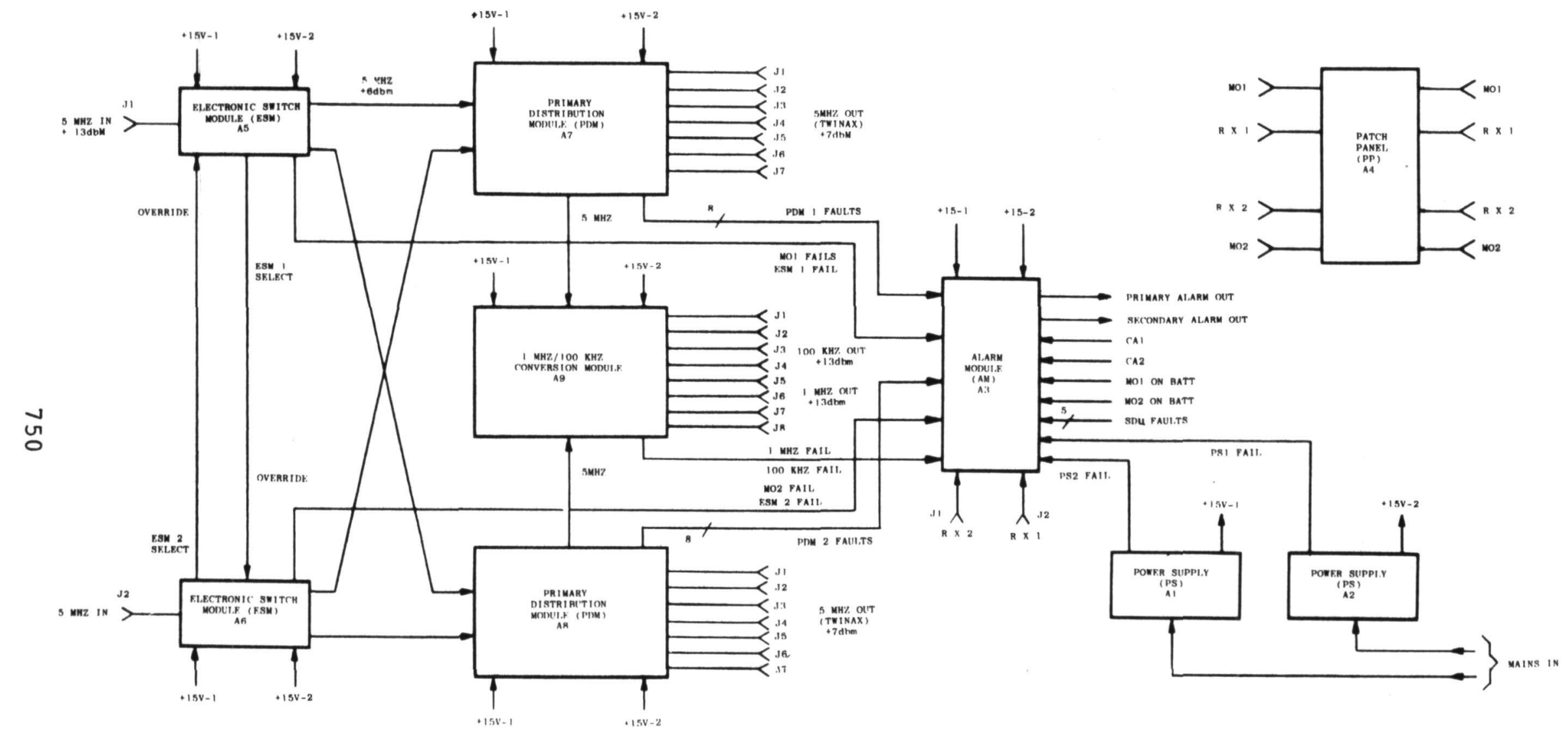

Figure 2. Primary Distribution Unit (PDU), Mode1 FE-798A, Block Diagram 


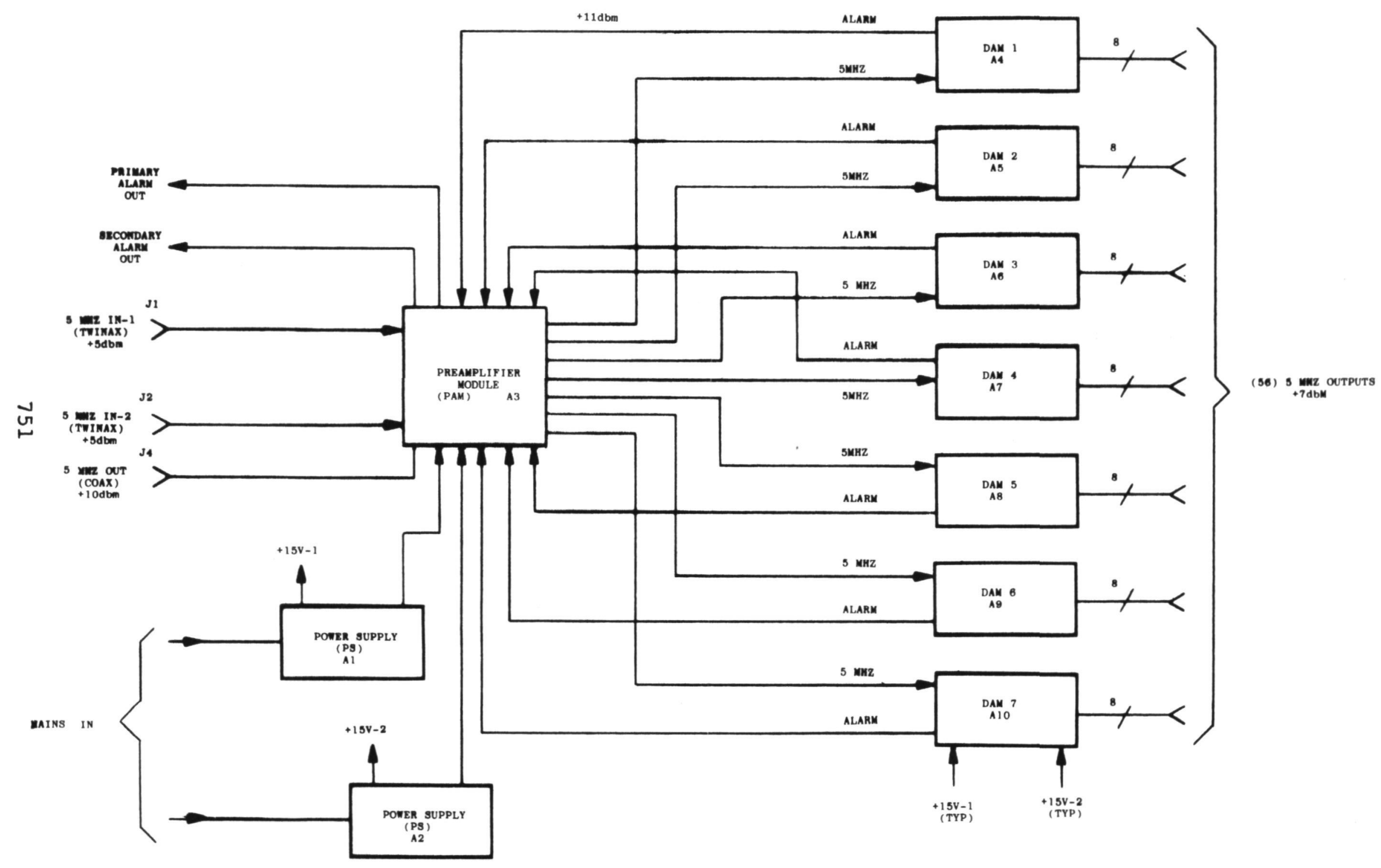

Figure 3. Secondary Distribution Unit (SDU), Mode1 FE-799A 


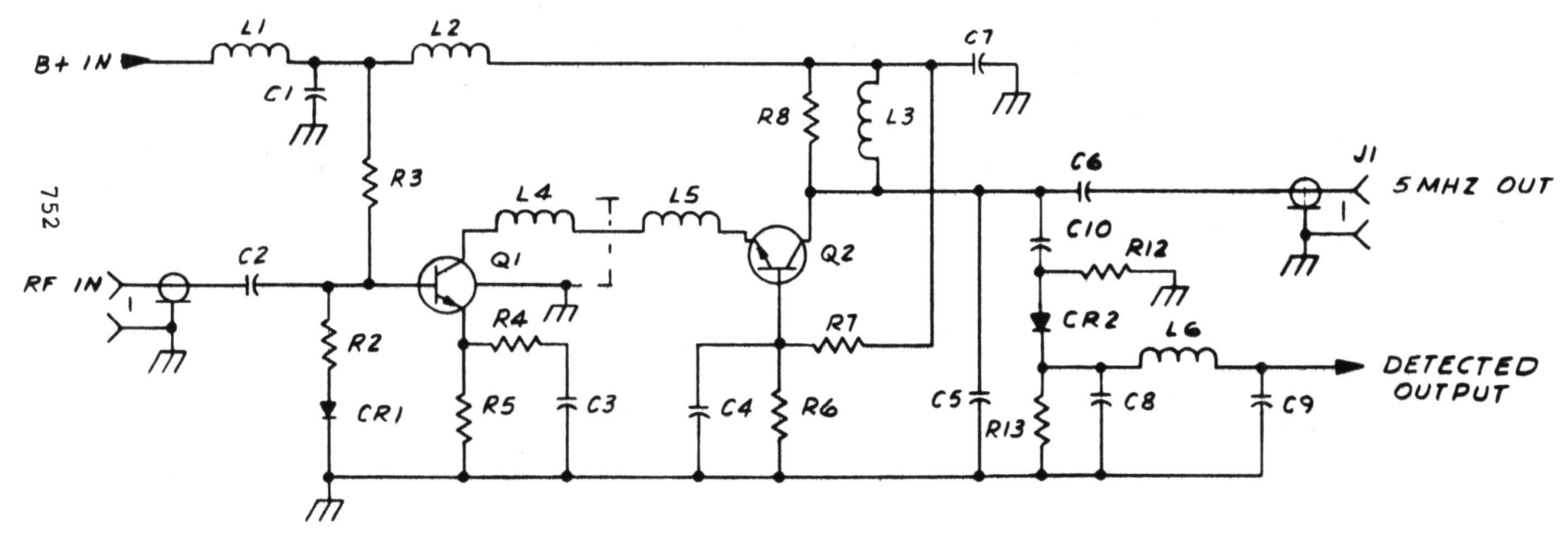

Figure 4. Cascode Amplifier, Schematic Diagram 


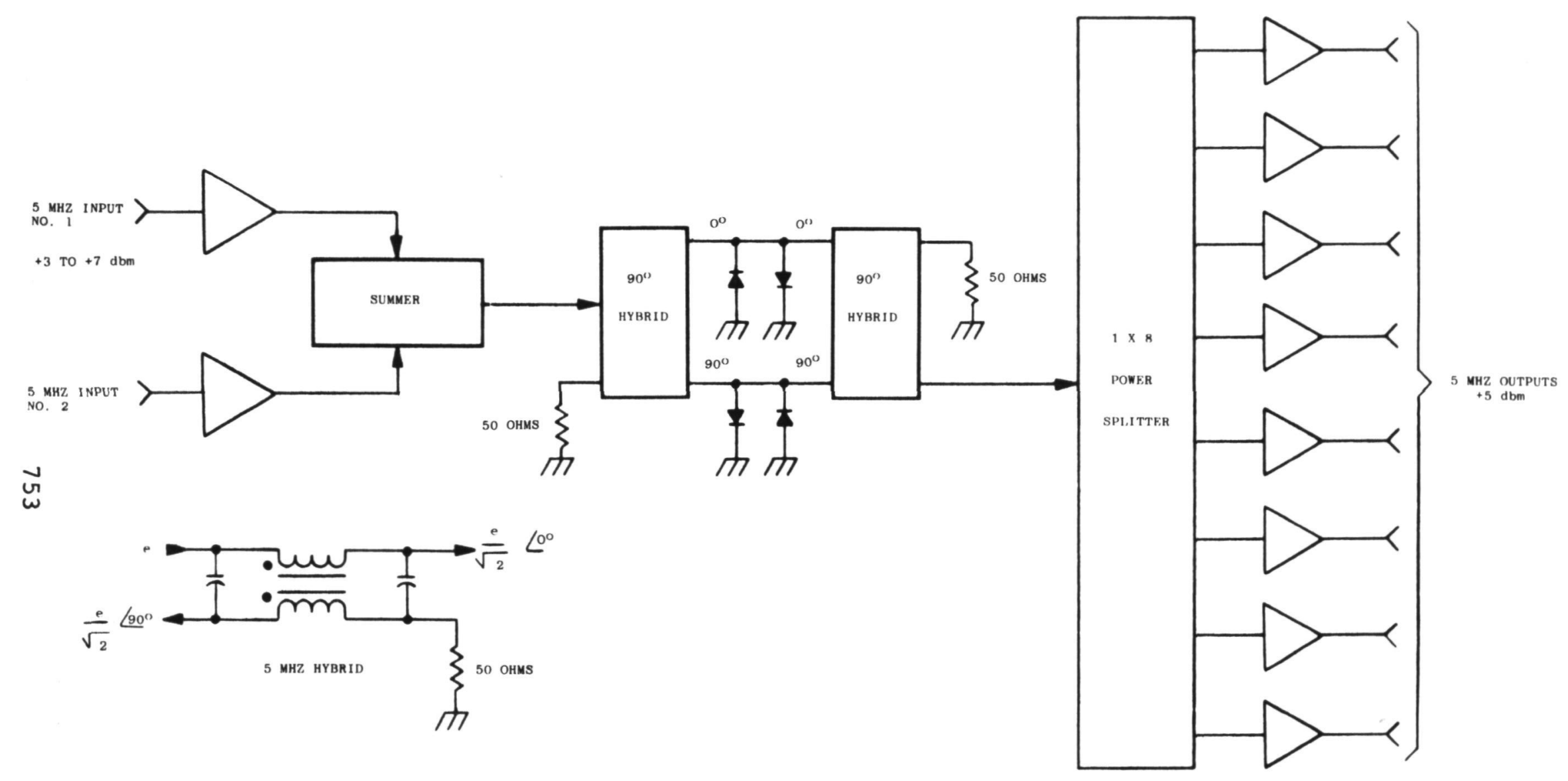

Figure 5. Block Diagram, Constant Impedance Limiter 


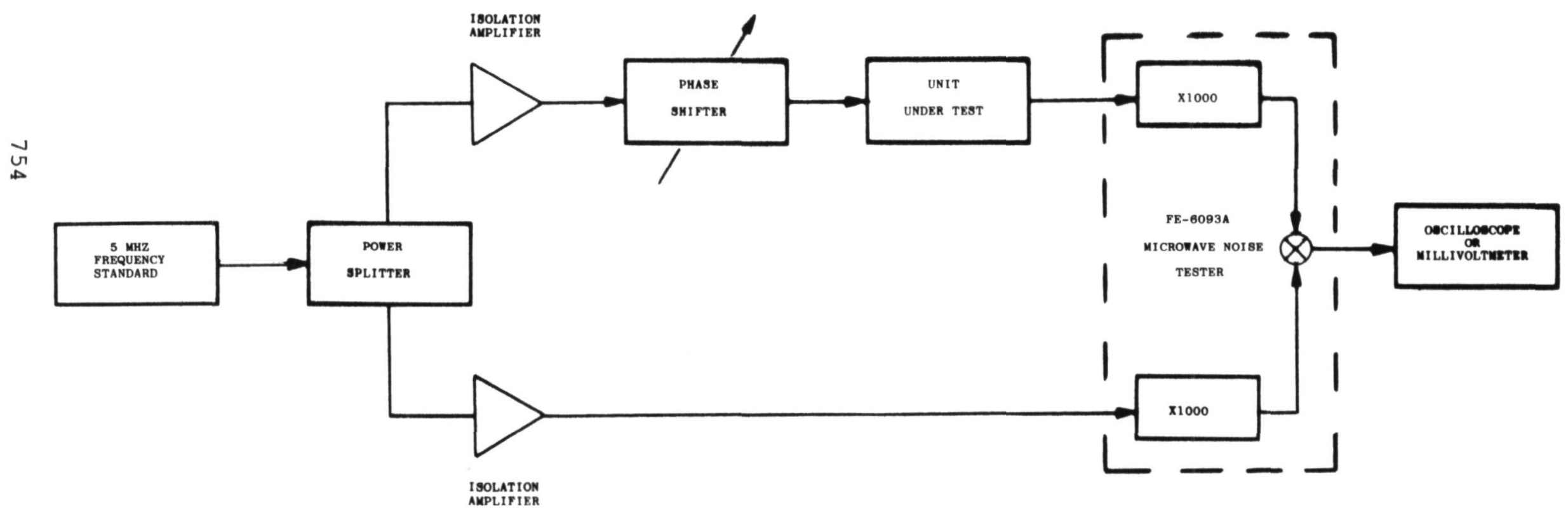

Figure 6. Test Set-Up, Phase Perturbations, Block Diagram 


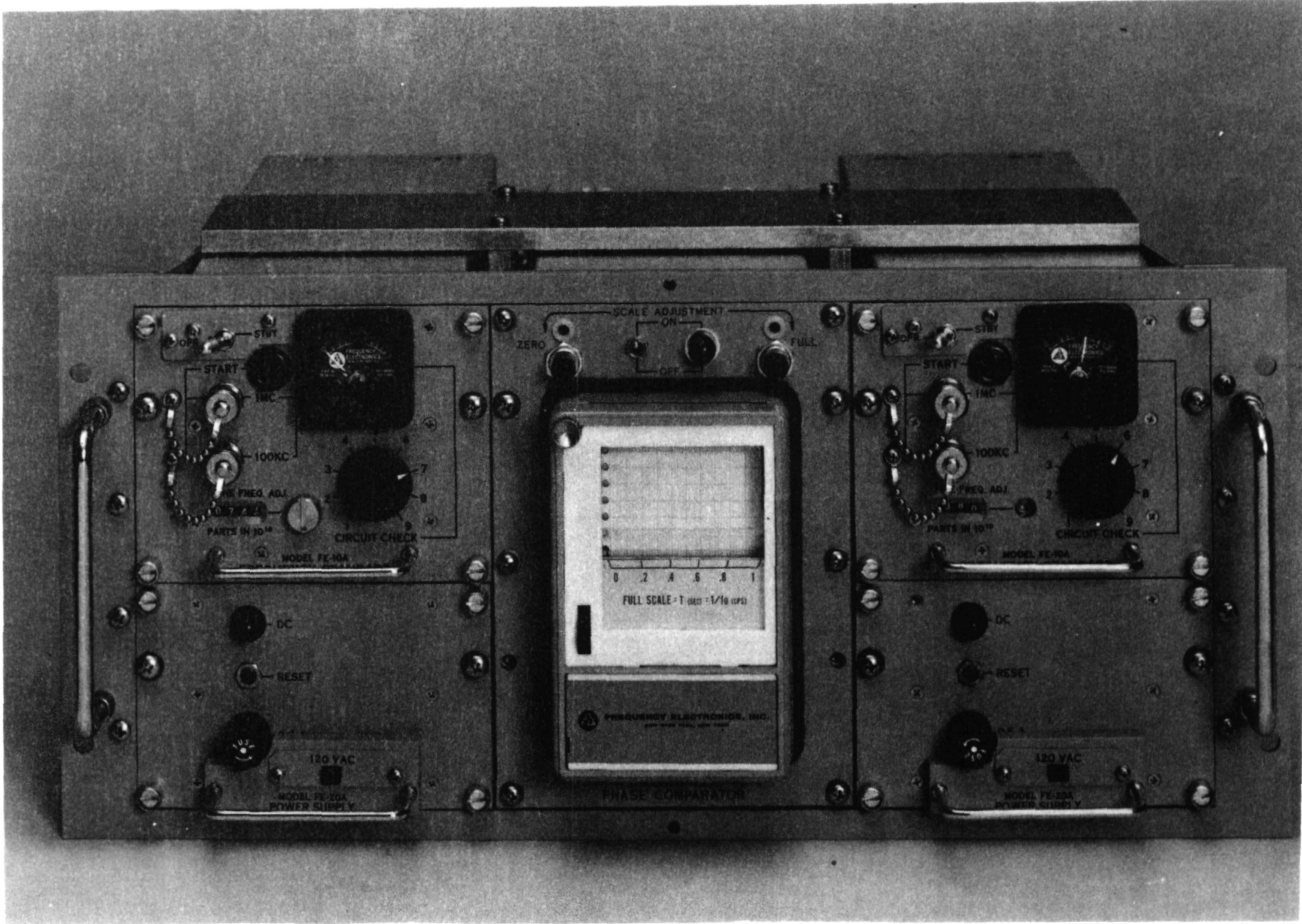

Figure 7. Frequency Generation Unit (FGU), Mode1 FE-5066A, 


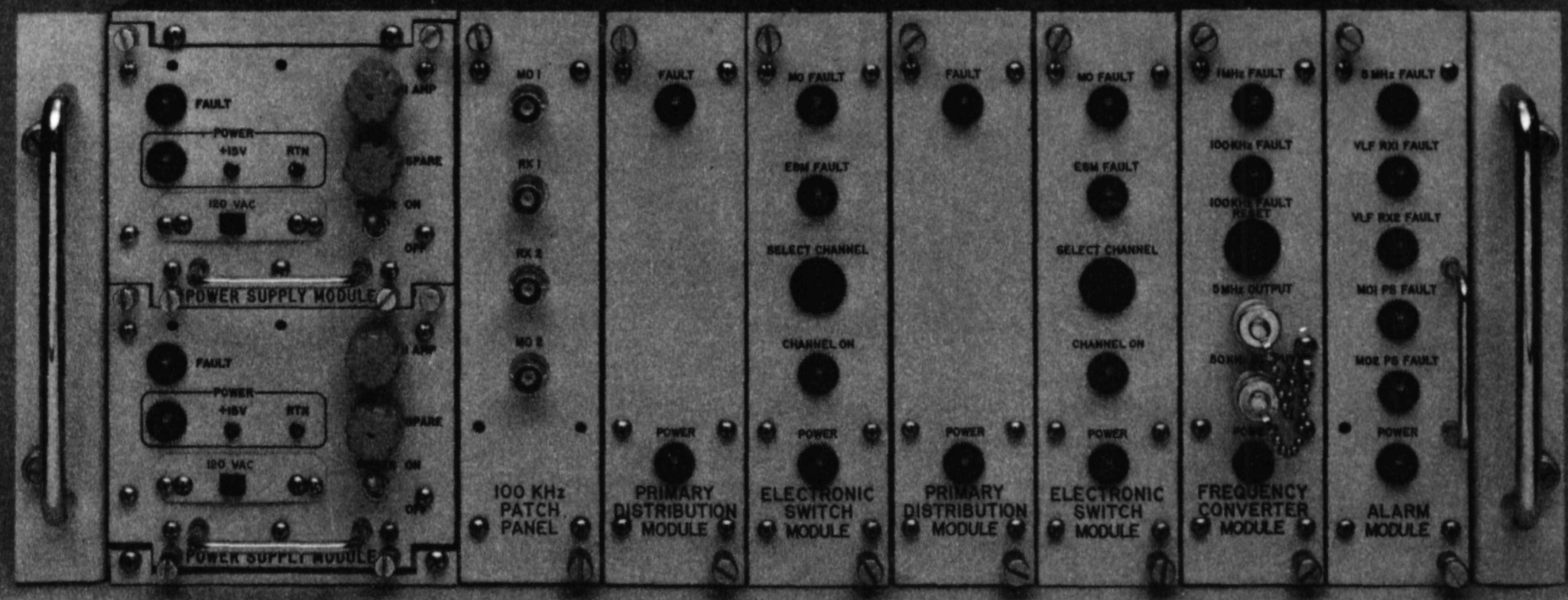

Figure 8. Primary Distribution Unit (PDU), Front View Mode1 FE-798A, 


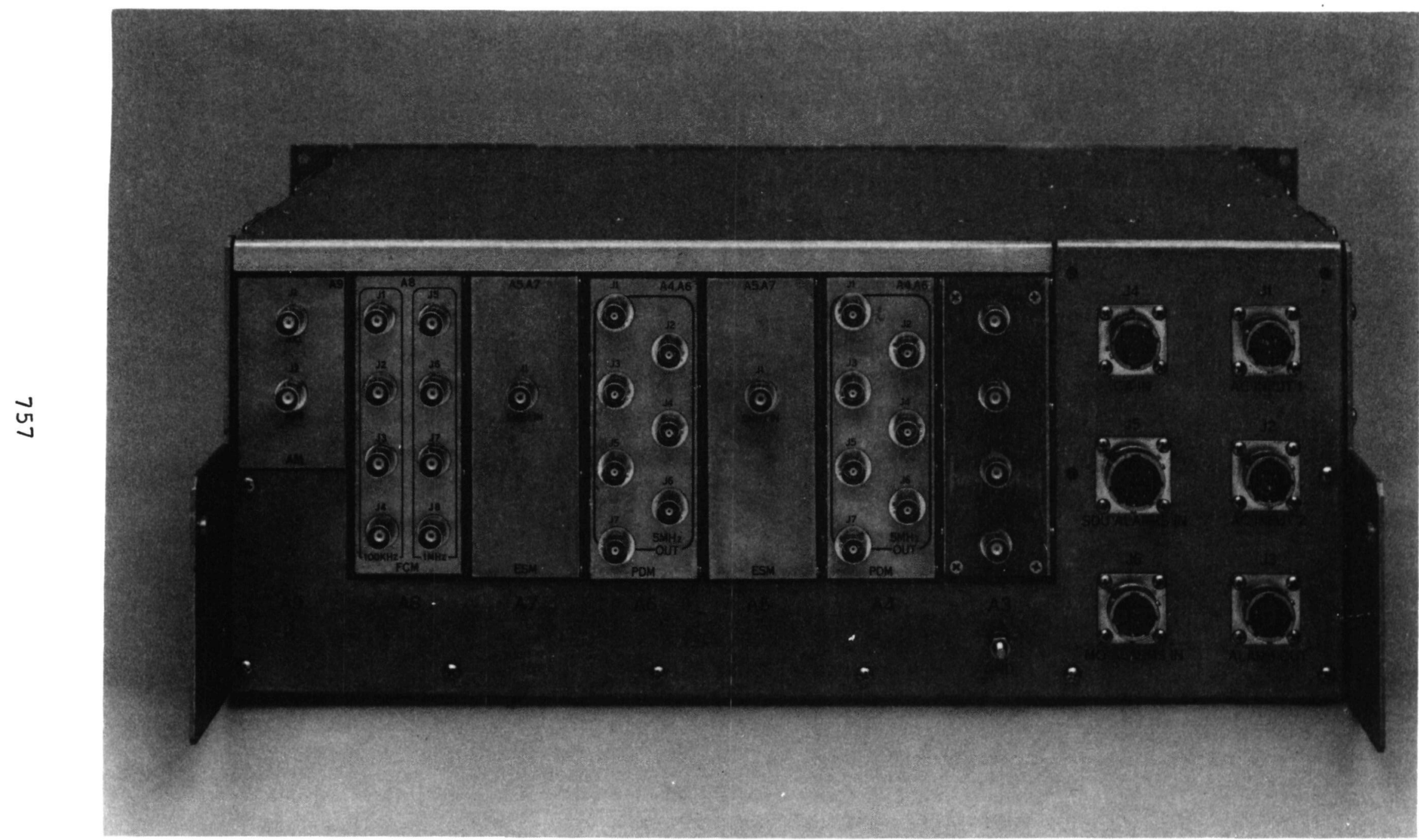

Figure 9. Primary Distribution Unit (PDU), Rear View Model FE-798A 


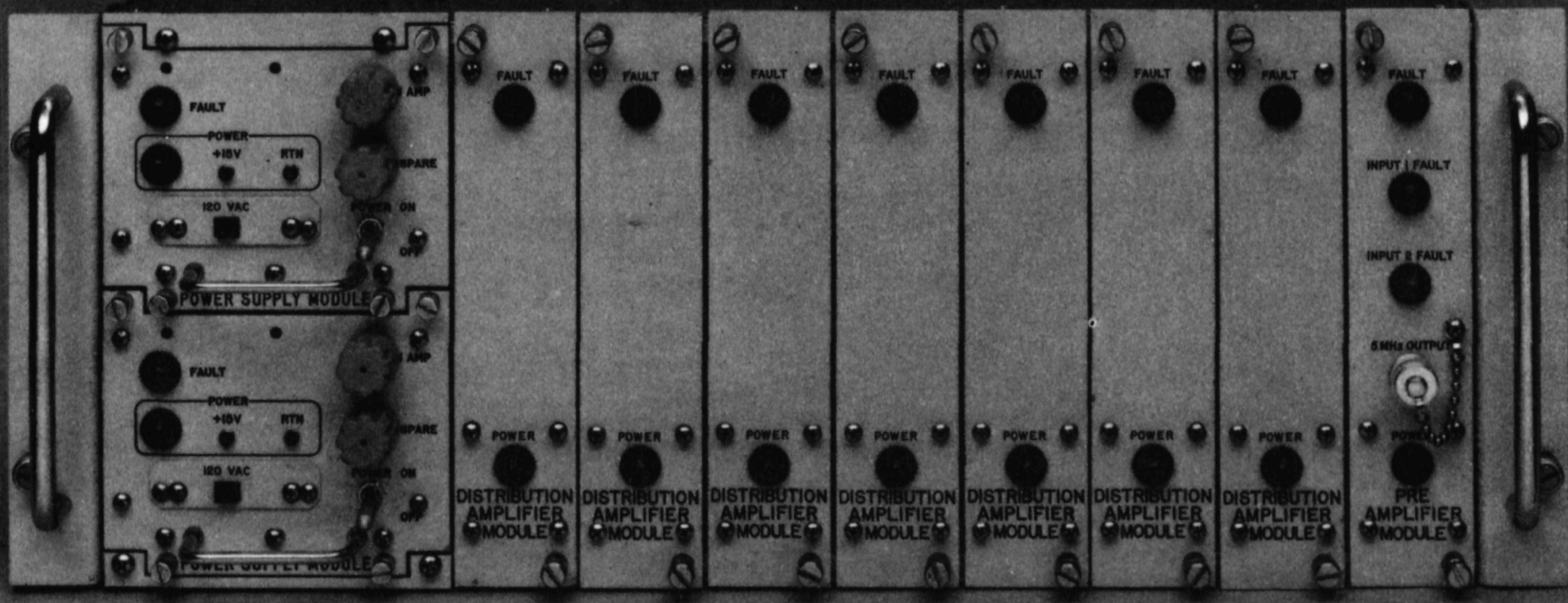

Figure 10. Secondary Distribution Unit (SDU), Front View Model FE-799A 


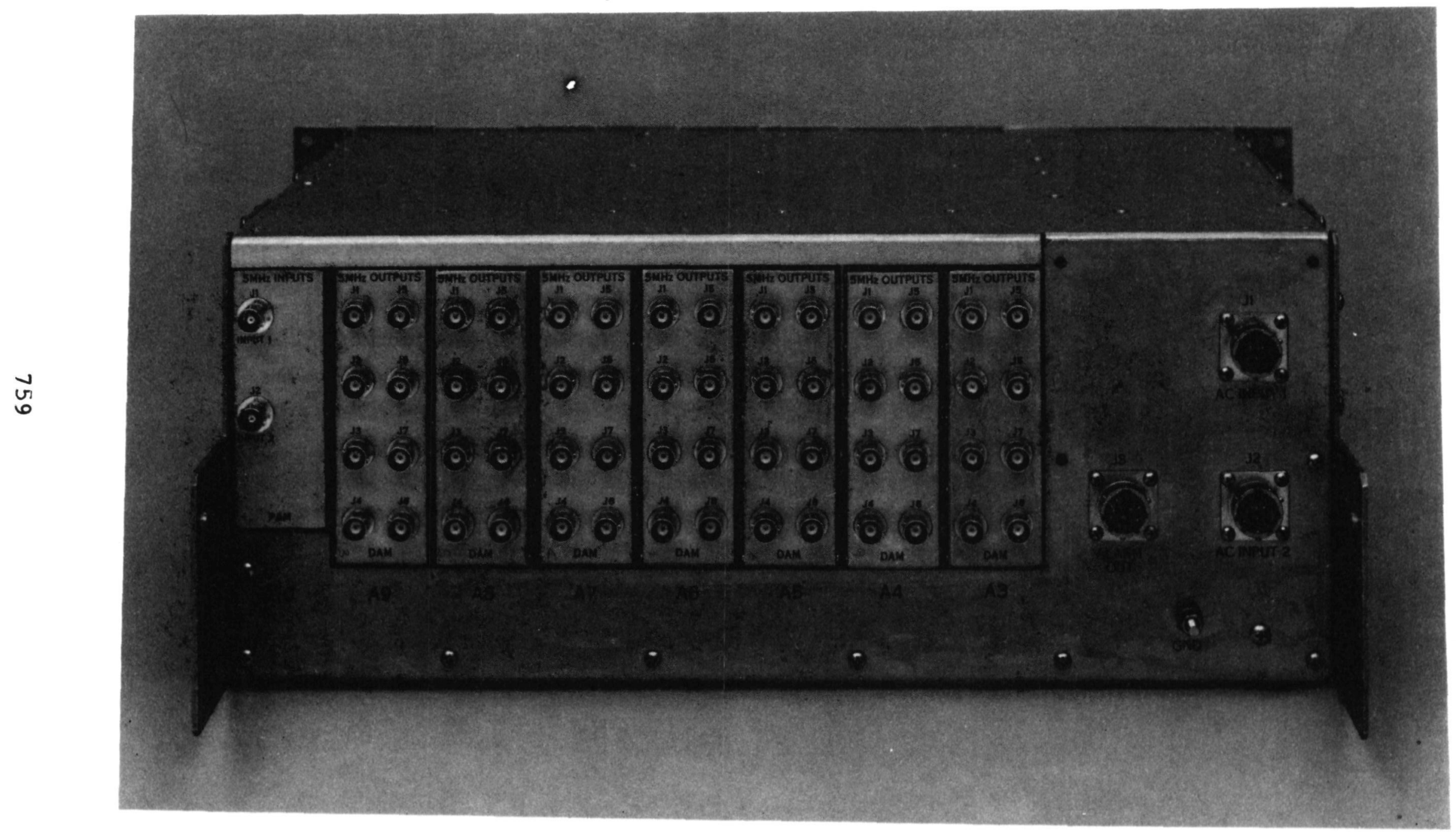

Figure 11. Secondary Distribution Unit (SDU), Rear View Mode1 FE-799A 


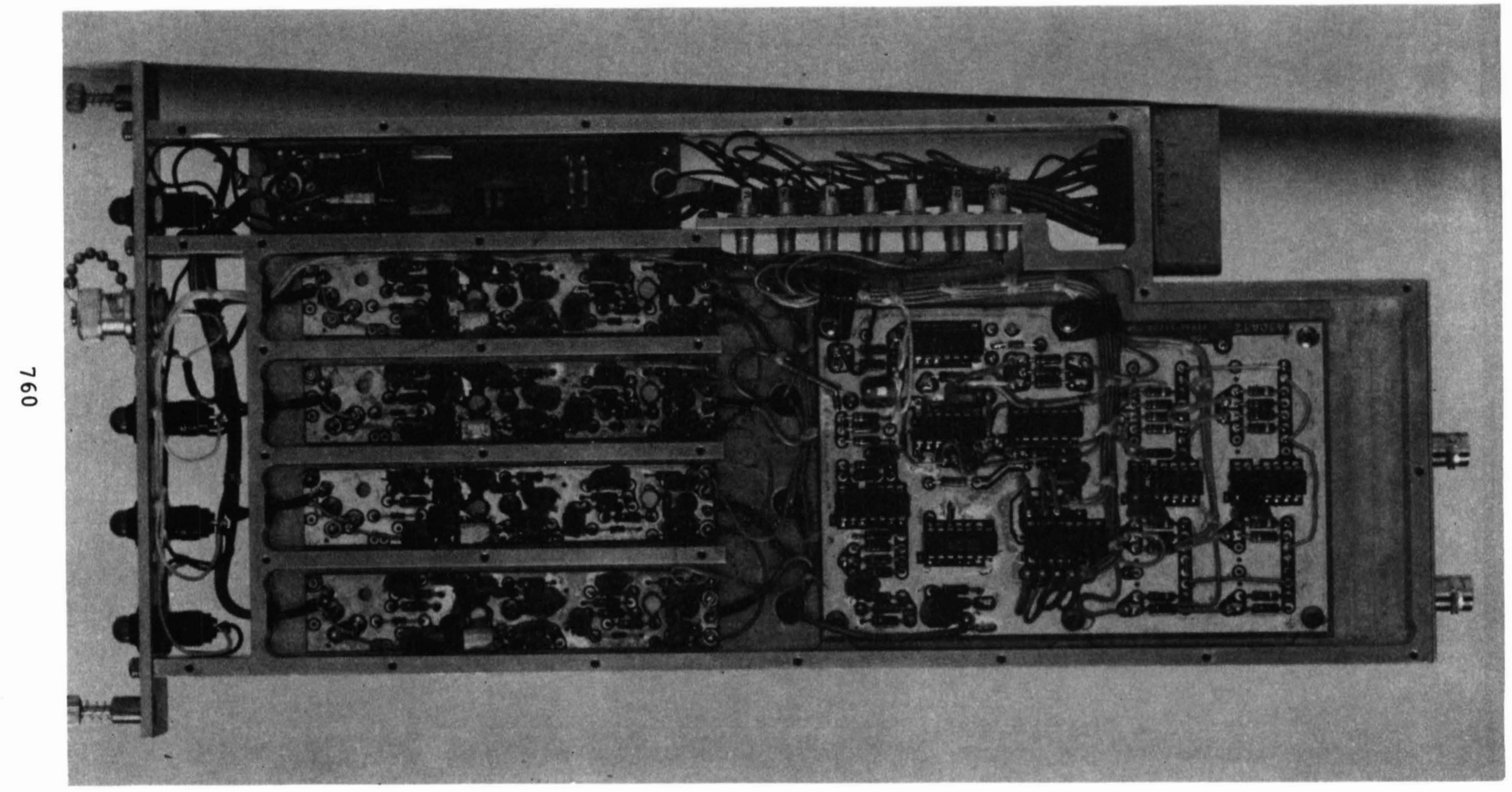

Figure 12. Distribution Amplifier Module, Top View 


\section{QUESTIONS AND ANSWERS}

MR. GEORGE PRICE, Austron

Marty, you said the frequency was being controlled by Loran-C? Is that true? Or was it VLF? I remember earlier that a NATO III was going to use VLF for frequency control.

MR. BLOCH :

It is LORAN. As a matter of fact, it is your receiver. Is that what you wanted to let me tell you, George?

MR. PRICE :

No, I thought the original plan was VLF, and I am surprised to see that it is LORAN-C.

MR. BLOCH:

We talked them out of it.

MR. PRICE :

Good.

MR. BLOCK:

But George, the basic concept involved is that LORAN is monitoring rather than driving, so in case there is any problem in an interruption, the system is still operational on the frequency standard. So that is a manual correction system that they chose.

MR. GEORGE LUTES, The Jet Propulsion Laboratory.

What is the magnitude of the amplitude to phase conversion? Did you say?

MR. BLOCH :

Are you taking in a limiter?

MR. LUTES:

Yes. 
MR. BLOCH :

It is a very good question. I can only give you a guesstimate. I would estimate that it adds to the noise floor about $4 \mathrm{~dB}$. So for optimum use, you are better off not using the limiter. There is no question about it; however, you can get $170 \mathrm{dBc}$, because the typical outputs over here are $+5 \mathrm{dBm}$ with the limiter, so anywhere from about 2 to $4 \mathrm{~dB}$ is added by the limiter -- if you had a ideal source driving it.

MR. LUTES :

If the amplitude of the signal changes by $10 \mathrm{~dB}$, for instance, how much phase change would that be in your system?

MR. BLOCH :

Al, did we measure that?

MR. VLUCAN:

It is one to two degrees.

MR. BLOCH :

It is a couple degrees at X-band over the whole dynamic range. The basic idea is that if you have a very good impedance match and the better your match the diode, you can theoretically have no phase shift at all. The phase shift is just a measure on the mismatch in the limiting diodes.

MR. LUTES:

Do you have any data on the phase change versus temperature?

MR. VULCAN :

No, but they are pretty broadband circuits.

MR. BLOCH:

What $A l$ is saying is that they are broadband circuits and he wouldn't expect much of a change over temperature. We will be testing them over the temperature range. Most of the requirements are short-term changes rather than long-term changes with temperature, because they don't want to unlock their up and down convertors. 
Taking a look at similar type of circuitry, my guesstimate would be that at X-band, it would probably be the equivalent of about two or three degrees over 0 to $50^{\circ} \mathrm{C}$ change. Or about two or three millidegrees at 5 megahertz.

MR. LUTES:

Thank you.

DR. STOVER, Defense Communications Engineering Center

In the first part of your talk where you are talking about all the redundance so that failures can occur without losing the signal, if you have a failure, can you detect and replace the failed part while you are still in service?

MR. BLOCH :

Absolutely. That was one of the major driving forces in this design: that you should be able to replace any and all modules and keep the equipment on line without disturbing the ones that have not failed. So when you pull in and out the power supply, there is less than a one millidegree shift in output phase and less than $.01 \mathrm{~dB}$ in amplitude. And if you have a 8-channel module that has failed, you can take that one out (again without disturbing the rest of the system) or one part of the electronic switch. That is why the electronic switch is not in one module. It is in two modules in order to have that type of feature.

You can take out the failed module without distrubing the rest of the system and put another tested module in, and your are back on line. And the faults are shown on the front panel as well as indicated remotely to a central monitoring point.

MR. P. BANERJEE, National Physical Laboratory, India

Actually, in India we are facing a very peculiar problem, because there is often power failure. And with the power failure on and off, you will find some high frequency surge which disturbs the system phase and all those things. I really want to know with your power supply, how much protection can it give against all these frequency surges?

MR. BLOCH:

Could you repeat the question? 
MR. HOWE:

He wants to know about protection and transients, I believe, on the power supply.

MR. BLOCH:

Are you talking about transients on the AC line?

MR. BANERJEE:

Yes.

MR. BLOCH :

This power supply has filtering and double regulations where large transients will (short-term transients in the millisecond range) have zero effect on the phase. There is no phase perturbation with fairly large short-term transient. And the input level can go from about 95 to 140 volts AC without affecting the output.

We are basically maintaining the voltage regulations of each module to about a millivolt. So it can take very large AC transients, because that is part of the problem that NATO III application has. They are normally on one power source and then they have a diesel backup and as soon as the power source fails it takes over to the diesel and there is a large transient that happens in this transition. So they have built in lots of transient and yet they must work through those transients. 